\title{
La muqueuse péri-implantaire : nécessité esthétique ou fonctionnelle ? Intérêt de l'augmentation de tissus mous peri-implantaires
}

\section{S. Aroca (Paris)}

L'anneau de tissu mou formé autour du col de l'implant est appelé muqueuse péri-implantaire. Un des rôles de cette attache tissulaire est de créer un joint biologique protecteur autour de l'implant afin de prévenir des maladies inflammatoires (mucosite, peri-implantite) qui pourraient compromettre l'ostéointegration.

Ce rôle protecteur est une évidence clinique malgré les controverses existantes dans la littérature en relation avec la nécessité de la présence ou absence des tissus kératinisés autour des implants. Mais quelle épaisseur tissulaire est capable d'assurer une stabilité du joint biologique péri-implantaire ? Le tissu conjonctif joue-t-il un rôle prépondérant? La stabilité du joint biologique, est-elle liée à l'épaisseur des tissus, à la stabilité des tissus osseux ou bien aux deux?

Par ailleurs, l'absence de muqueuse kératinisée, un phenotype fin, aussi une insuffisance de volume, tant des situations cliniques qui risquent de favoriser l'instabilité des tissus péri-implantaires.

Les conséquences cliniques (outre le risque de maladie inflammatoire) sont multiples :

1. Mobilité et ou décollement de la muqueuse péri-implantaire

2. Récession de la muqueuse péri-implantaire

3. Restauration implanto-portée inesthétique par rapport aux dents adjacentes

Cet exposé a pour but de répondre à toutes ces questions et aussi de détailler les solutions qui sont nécessaires pour assurer ou rétablir une stabilité de la muqueuse péri-implantaire. 